\title{
Next Generation Sequencing and Association Studies in Familial Nonmedullary Thyroid Carcinoma: Let's Choose Appropriate Controls
}

\author{
Laure Cazabat ${ }^{a}$ b Aglae Terray $^{a}$ Philippe de Mazancourt ${ }^{c}$ Jacques Ropers ${ }^{d}$ \\ Lionel Groussin ${ }^{e, f}$ Marie-Laure Raffin-Sanson ${ }^{a, b}$

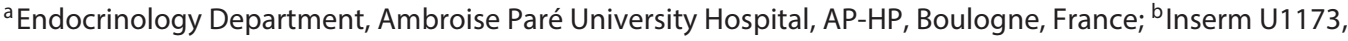 \\ Université Versailles-Saint-Quentin, Montigny-Le-Bretonneux, France; ' UMR U1179, U1173, Université \\ Versailles-Saint-Quentin, Montigny-Le-Bretonneux, France; ${ }^{\mathrm{d}}$ Clinical Research Unit, Ambroise Paré University \\ Hospital, AP-HP, Boulogne, France; ${ }^{e}$ Department of Endocrinology, Cochin University Hospital, AP-HP,

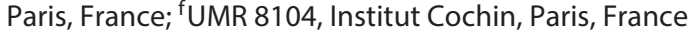

Dear Editor,

Familial nonmedullary thyroid cancer (FNMTC) is clinically defined by the presence of thyroid cancer (papillary or follicular) in two or more first-degree relatives in the absence of predisposing environmental factors. This entity accounts for 3 to $9 \%$ of all cases of thyroid cancer. Among FNMTC, 5\% are syndromic forms with a well-defined germline mutation whereas $95 \%$ are nonsyndromic with a less clear genetic predisposition. Previous linkage analysis failed to point out a main locus responsible for the genetic predisposition to FNMTC even if several loci have been pointed out [1]. More than $90 \%$ of thyroid cancers are sporadic, secondary to somatic alterations.

Using next generation sequencing, Gara and Kebebew [2] reported a germline variant (rs7080536, responsible for a p.G534E substitution) in $H A B P 2$ (encoding hyaluronan-binding protein, also named factor VII-activating protease) associated with papillary thyroid cancer (PTC) in a large kindred: PTC in 6 patients and follicular adenoma in 1 subject. Genetically related unaffected members were not available for testing. Moreover, based on the difference of frequencies of the G534E variant in a series of 423 PTC from The Cancer Genome Atlas (TCGA) database $(4.7 \%)$ in comparison with $0.7 \%$ in the 1000 Genomes database used as a control group, $H A B P 2$ was also proposed as a susceptibility gene in sporadic PTC tumors. In vivo studies came to comfort the role of HABP2 in thyroid tumorigenesis [2].

Noteworthy, Gara and Kebebew [2] considered the HABP2 variant as a candidate for further analysis because the frequency of the rs7080536 minor allele was under $1 \%$ in the HapMap 3 and $1000 \mathrm{Ge}$ nomes databases used by the authors as reference populations for the analysis of whole exome data. However, the frequency of rs7080536 was reported to be between 4 and 6\% in some European populations (Table 1). Such frequencies arise from previous studies describing the G534E variant (rs7080536; Marburg variant) implication in the predisposition to cardiovascular disease. Also, the HABP2 G534E frequency in the control population used by Gara and Kebebew, i.e. the
1000 Genomes database, was quite low $(0.7 \%)$ compared with other public databases such as the EXome Aggregation Consortium database (2.2\%, Table 1$)$.

Since this report, emerging research has failed to reproduce these results, either in familial or in sporadic forms of nonmedullary thyroid cancer (NMTC) in different populations of patients [3-7], among them three European studies [8-10] (Table 2). These studies are based on the comparison of frequencies of the variant in patients with NMTC versus controls chosen in populations from the same geographic areas. Moreover, in familial studies, the HABP2 G534E variant fails to segregate with the disease [4, $7,9]$. These results cast doubt on the role of HABP2 G534E in the predisposition to PTC.

Some data however remain unexplained. Gara and Kebebew [2] provided functional demonstration of the tumorsuppressive effect of HABP2: overexpression of wild-type HABP2 in thyroid and nonthyroid cell lines reduced colony formation and cell migration. Overexpression of the G534E variant increased colony formation. Tomsic et al. [4] pointed out the

\section{KARGER}

E-Mail karger@karger.com www.karger.com/etj
C 2017 European Thyroid Association

Published by S. Karger AG, Basel
Marie-Laure Raffin-Sanson Hôpital Ambroise Paré 9 Avenue Charles de Gaulle FR-92100 Boulogne (France)

E-Mail marie-laure.raffin-sanson@aphp.fr 
Table 1. HABP2 G534 frequency in patients with sporadic NMTC and control subjects from published reports and public databases

HABP2 G534E frequency

Miscellaneous origin

1000 Genomes project phase $1^{\circ}(A)$

1000 Genomes project phase $3^{\circ}(A)$

${ }^{\circ}$ Europe: $14 \%$, North America: $8.5 \%$, Asia: 33\%,

Africa: 29\%, South America $15.5 \%$

ExAC Database (all) (C)

\section{Asia \\ ExAC Database: South Asia (C) \\ ExAC Database: East Asia $(C)$}

Controls from Saudi Arabia, Middle East [5]

\section{Africa}

ExAC Database: African (C)

$0.54 \%(n=5,203)$

\section{South America}

Hispanic controls (Columbia) [6]

ExAC Database: Hispanic (C)

\section{North America}

NHLBI Grand Opportunity Genome Seq Project (D)

European Americans/African Americans

Controls USA, Ohio cohort, mainly Caucasian [4]

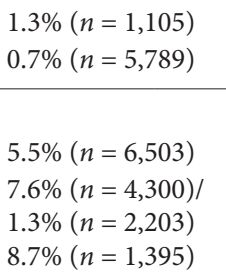

$88 \%(n=8,256)$
$0 \%(n=4,327)$

NMTC, Beijing (China) [3]

NMTC, Saudi Arabia [5]

$0.5 \%(n=190)$
$7.8 \%(n=1,160)$
NMTC, 5 Columbian cities [6]

\section{Australia}

The 1994-1995 Busselton Health Study [7]

$7.6 \%(n=4,634)$

\section{Europe}

Bruneck Study, Italy [12]

Blood donors, Germany [13]

PROSPER study, UK, Netherlands [14]

GENDER study, Netherlands [14]

ExAC Database: European (C)

UK controls, 1958 Birth Cohort [8]

Spanish controls [9]

$0 \%(n=217)$

$0.2 \%(n=509)$

\section{$4.7 \%(n=423)$}

$0.2 \%(n=509)$

TCGA, worldwide* (B)

Ancestry: unknown: $18.5 \%$, Asian: $10.3 \%$, African 5.3\%, White American: $65.7 \%$

Present series

$$
\begin{gathered}
4.4 \%(n=810) \\
2.3 \%(n=213) \\
5 \%(n=5,804) \\
4 \%(n=3,104) \\
3.3 \%(n=33,370) \\
4.6 \%(n=5,172) \\
2.24 \%(n=267)
\end{gathered}
$$
USA [4]

$4.9 \%(n=203)$
$6.3 \%(n=143) /$
$0 \%(n=45)$

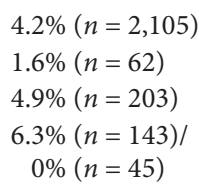

\author{
TCUKIN cases: NMTC, UK [8] \\ Juvenile Spanish NMTC patients [9] \\ French NMTC patients, \\ from European ancestry/from African \\ ancestry
}

(A), www.1000genomes.org; (B), http://cancergenome.nih.gov/; (C), www.exac.broadinstitute.org; (D), http://evs.gs.washington.edu/; NMTC, nonmedullary thyroid cancer; PTC, papillary thyroid cancer; TCGA, The Cancer Genome Atlas. Numbers in square brackets refer to reference numbers printed at the end of the text. Frequencies used by Gara and Kebebew [2] are in white characters on black background.

faint expression of HABP2 in thyroid tissues at the RNA level: qPCR detected no $H A B P 2$ mRNA expression in tumoral tissue from sporadic PTCs or adjacent normal tissue, in patients that either carry or do not carry the variant. However, the dissociation between the mRNA and protein levels in several tissues among them thyroid tissues suggests posttranscriptional and posttranslational regulation of HABP2.
Recently, another report provided new data supporting the role of HABP2 G534E as a susceptibility factor in some families with PTC: among 43 subjects with FNMTC from 29 relatives, the overall prevalence of HABP2 G534E was 14\% (6/43). None of the subjects with benign neoplasm or normal subjects from these families had this variant [11]. Thus, the question remains open to debate.
In this context, we wished to contribute to the clarification of the role of HABP2 in thyroid cancer in studying the frequency of the G534E variant in a third European series of 203 patients with sporadic NMTC, in which the ancestry is known. Since Gara and Kebebew [2] considered as "affected member" 1 subject with a follicular adenoma, we also analyzed 99 patients with nonmalignant follicular adenomas. We ana- 
Table 2. HABP2 G534 frequency in patients with familial NMTC from published reports

\begin{tabular}{lll}
\hline Patients (familial cases) & $\begin{array}{l}\text { Frequency of the HABP2 } \\
\text { G534E variant }\end{array}$ & $\begin{array}{l}\text { Segregation } \\
\text { with the disease }\end{array}$ \\
\hline 1 family (7 subjects) [2] & $\begin{array}{l}\text { present in all affected } \\
\text { members }\end{array}$ & yes \\
\hline 12 probands from families with PTC (Beijing, China) [3] & $0 \%$ & NA \\
\hline $\begin{array}{l}103 \text { families with } 3 \text { cases of PTC or more, Ohio cohort } \\
\text { (mainly Caucasian) [4] }\end{array}$ & $11 / 179(6.1 \%)$ & no \\
\hline \begin{tabular}{l} 
a cases from 29 families with PTC (USA) [11] \\
\hline 11 subjects from 4 families with NMTC (Saudi Arabia) [5]
\end{tabular} & $0 \%$ & NA \\
\hline $\begin{array}{l}16 \text { Spanish families with NMTC, 33 affected/44 unaffected } \\
\text { members [9] }\end{array}$ & $2 / 77(2.6 \%)$ & no ${ }^{\text {a }}$ \\
\hline $\begin{array}{l}\text { 63 probands from Italian families with PTC and } 41 \\
\text { unaffected members [10] }\end{array}$ & $0 \%$ & NA \\
\hline
\end{tabular}

Numbers in square brackets refer to reference numbers printed at the end of the text. NMTC, nonmedullary thyroid cancer; PTC, papillary thyroid cancer; NA, not available. ${ }^{\text {a }}$ In the same family, affected members may or may not have the HABP2 G543 variant.

lyzed in all these subjects the presence or the absence of a multinodular goiter in relation to the $H A B P 2$ genotype.

Two hundred and three adult patients (mean: 48.96 years [range: $18-78]$, female: $n=161$, male: $n=42$ ) with histologically proven NMTC (papillary carcinoma: $n=$ 174, follicular carcinoma: $n=29$ ) were recruited in three Parisian hospitals. Clinical, echographic, and histological data were collected from their medical record and from face-to-face interviews. Patients gave written informed consent before undergoing evaluation and blood sampling. The ethical committee of the Cochin Hospital approved the study. PCR amplification was carried out with mutagenic primers (TGTAAAACGACGGCCAGT-AGCTGGGGCCAGGAGTGTG and 6-FAMGAGAGTGGGCTCTGAGGTCCAG). The underlined mutation induces a BstXI cleavage site on the $G$ allele (coding for p.G534). An M13 sequence (in italics) was added on the forward primer to improve the resolution. An FGG fragment (GGATCTGGTTGGTGGATGAACA and 6-FAM-GGGCTAATTTTAGGCATGAACTTGGTAT) with a mandatory BstXI site was co-amplified as a control. We also revisited the published data about $H A B P 2$ genotypes in different populations of normal subjects and in patients with NMTC in the light of the geographical origin of the subjects.

We noted the presence of $H A B P 2$ G534E in $10 / 203$ patients with NMTC from France $(4.9 \%)$ and in $8 / 133(6.0 \%$, $95 \% \mathrm{CI}=2.6 \% ; 11.5 \%)$ when considering only the patients of European descent. This latter frequency is not significantly different from those observed in European subjects in public databases, i.e. 3.3\% in the ExAC database and $7.6 \%$ in the NHLBI Grand Opportunity Genome Seq Project (Table 1). Among 99 patients with follicular adenomas, 5 had the polymorphism (5\%). We observed no significant difference of $H A B P 2$ G534E frequency between patients with multinodular goiter $(6.8 \%$ $[11 / 162])$ and patients without multinodular goiter (2.9\% [4/140]; $p=0.18$, Fisher's exact test). However, the confidence interval of the odds ratio is wide and would need a larger sample to draw a definitive conclusion $(\mathrm{OR}=2.5,95 \% \mathrm{CI}=0.7 ; 10.9)$.

Among the 10 patients carrying the G534 allele (8 females, 2 males), all had papillary tumors. We observed no substantial difference in the clinical presentation and histological data between patients carrying or not carrying the G534 allele: mean age at diagnosis was 54.6 years (SD: 18.4) versus 48.7 years (SD: 16.4) $(p=0.27$, Wilcoxon rank-sum test), 3/9 (33\%) had extrathyroid invasion versus $102 / 186(55 \%)$ of patients who did not carry the variant ( $p=$ 0.31 , Fisher's exact test), 5/9 (55\%) had multifocal tumors versus $68 / 184(37 \%)$ in the absence of the variant $(p=0.30)$, and $2 / 9$ (22\%) had lymph node invasion versus $66 / 185(36 \%)$ of patients not carrying the variant $(p=0.50)$. Thus, in our series the presence of the allele did not substantially influence the presentation of the tumor.

Analysis of data from public databases and previously published studies clearly indicates a difference in the frequency of the G534E variant depending on the continent and whether patients with sporadic NMTC or controls are studied. The lowest frequency of the variant is observed in Asian and African populations ( 0 to $0.88 \%$ ). The highest frequency is reported in Europeans (2.25 to 5\%) and North American populations of European descent (7.6 to 8.7\%). Populations from South America (Hispanic) had an intermediate frequency ( 0.7 to $1.3 \%$ ) (Table 1). In each population group, the frequency of the variant was similar in patients with sporadic PTC and normal controls of the same descent.

The reason why the frequency of the G534E variant which emerged was differ- 
ent in the two miscellaneous populations tested by Gara and Kebebew [2], i.e. patients with PTC from the TCGA database and the control groups (1000 Genomes project), may be explained by the different ancestry proportions and their different frequencies of G534E carrying in the two populations. In patients with PTC from the TGA database, the proportion of Asian and African subjects (in whom G534E frequency is close to $0 \%$ ) represents $15.6 \%$ of the total versus $62 \%$ in the 1000 Genomes project, chosen as the control group (Table 1). The ethnicity of the family reported by Gara and Kebebew is unknown, but, if it were of European descent, the rs7080536 variant would not have passed the filter criterion during analysis of the next generation sequencing data with an appropriate control population.

\section{References}

1 Ngeow J, Eng C: HABP2 in familial non-medullary thyroid cancer: will the real mutation please stand up? J Natl Cancer Inst 2016; 108:djw013.

-2 Gara SK, Kebebew E: HABP2 mutation and nonmedullary thyroid Cancer. N Engl J Med 2015;373:2086-2087.

3 Zhao X, Li X, Zhang X: HABP2 mutation and nonmedullary thyroid cancer. N Engl J Med 2015;373:2084.

-4 Tomsic J, Fultz R, Liyanarachchi S, He H, Senter L, de la Chapelle A: HABP2 G534E variant in papillary thyroid carcinoma. PLoS One 2016;11:e0146315.

-5 Alzahrani AS, Murugan AK, Qasem E, AlHindi H: HABP2 gene mutations do not cause familial or sporadic non-medullary thyroid cancer in a highly inbred Middle Eastern population. Thyroid 2016;26:667-671.

6 Bohórquez ME, Estrada AP, Stultz J, Sahasrabudhe R, Williamson J, Lott $\mathrm{P}$, Duque CS, Donado J, Mateus G, Bolaños F, Vélez A, Ech-
Noteworthy, if the variant does not seem to predispose individuals to NMTC when accounting for geographical ancestry, this does not mean that the protein cannot promote thyroid cancer progression or dissemination. According to its role in endothelial barrier modulation, HABP2 could indeed facilitate thyroid cancer invasiveness as proposed for lung cancer. This is reminiscent of the association of rs7080536 with cardiovascular events, possibly via modulation of hemostasis and vascular remodeling processes.

In conclusion, we confirm with this study in a European population of adults with either NMTC or follicular adenoma or GMN a similar frequency of the G534E variant in patients and in subjects without thyroid tumor of the same ancestry. We underline the different frequency of the polymorphism in subjects of European or non-European descent. This emphasizes the importance of matching the patients with controls from appropriate databases with a similar ancestry composition.

\section{Disclosure Statement}

The authors declare that they have no competing interests.

\section{Funding Sources}

This work was supported by Assistance Publique Hôpitaux de Paris funding and a grant from the Direction Générale de l'Offre de Soin: PHRC AOR01093. everry M, Carvajal-Carmona LG: The HABP2 G534E polymorphism does not increase nonmedullary thyroid cancer risk in Hispanics. Endocr Connect 2016;5:123-127.

-7 Weeks AL, Wilson SG, Ward L, Goldblatt J, Hui J, Walsh JP: HABP2 germline variants are uncommon in familial nonmedullary thyroid cancer. BMC Med Genet 2016;17:60.

-8 Sahasrabudhe R, Stultz J, Williamson J, Lott P, Estrada A, Bohorquez M, Palles C, Polanco-Echeverry G, Jaeger E, Martin L, Magdalena Echeverry M, Tomlinson I, CarvajalCarmona LG; TCUKIN: The HABP2 G534E variant is an unlikely cause of familial nonmedullary thyroid cancer. J Clin Endocrinol Metab 2015;jc20153928.

-9 Ruiz-Ferrer M, Fernández RM, Navarro E, Antiñolo G, Borrego S: G534E variant in HABP2 and nonmedullary thyroid cancer. Thyroid 2016;26:987-988.

10 Cantara S, Marzocchi C, Castagna MG, Pacini F: HABP2 G534E variation in familial non- medullary thyroid cancer: an Italian series. J Endocrinol Invest 2017;40:557-560.

11 Zhang T, Xing M: HABP2 G534E mutation in familial nonmedullary thyroid cancer. J Natl Cancer Inst 2016;108:djv415.

12 Willeit J, Kiechl S, Weimer T, Mair A, Santer P, Wiedermann CJ, Roemisch J: Marburg I polymorphism of factor VII-activating protease: a prominent risk predictor of carotid stenosis. Circulation 2003;107:667-670.

13 Hoppe B, Tolou F, Radtke H, Kiesewetter H, Dörner T, Salama A: Marburg I polymorphism of factor VII-activating protease is associated with idiopathic venous thromboembolism. Blood 2005;105:1549-1551.

14 Trompet S, Pons D, Kanse SM, de Craen AJ, Ikram MA, Verschuren JJ, Zwinderman AH, Doevendans PA, Tio RA, de Winter RJ, Slagboom PE, Westendorp RG, Jukema JW: Factor VII activating protease polymorphism (G534E) is associated with increased risk for stroke and mortality. Stroke Res Treat 2011;2011:424759. 